\title{
Evaluation of a protocol for selective empiric treatment of fever without localising signs
}

\author{
G J Browne, J M Ryan, P McIntyre
}

\begin{abstract}
A protocol for management of young febrile children at risk for bacteraemia has been used at Westmead Hospital, a university based hospital in the western Sydney region, since early 1994. Implementation of the protocol was retrospectively evaluated for the 12 month period 1 June 1994 to 31 May 1995, using the emergency department log book as the primary data source. Altogether 498 children, aged from 3 months to 3 years, with a fever $\geqslant 39.5^{\circ} \mathrm{C}$ were identified over this period, of whom 291 were admitted to hospital because of evidence of sepsis or identified focal infection and 207 children without focal infection were observed in the short stay annexe of the emergency department. Fifty children, considered at high risk of bacteraemia because of a total white cell count $\geqslant 20 \times 10^{9} / 1$ received empiric antibiotic treatment with ceftriaxone, of whom 19 subsequently had proved bacteraemia and another 10 had focal infection identified during observation in the short stay annexe. Bacteraemia was due to Streptococcus pneumoniae in 16 cases and Haemophilus influenzae type $b$ in three. No adverse events occurred at follow up.

Use of a management protocol and selection on higher white cell count criterion than previously recommended by US centres resulted in restriction of empiric antibiotic treatment to a small proportion of young febrile children presenting to a busy emergency department of whom $38 \%$ were bacteraemic.

(Arch Dis Child 1997;76:129-133)
\end{abstract}

Keywords: bacteraemia; ceftriaxone; emergency department.

Westmead Hospital,

Westmead, Australia:

Emergency

Department

G J Browne

J M Ryan

Department of

Paediatrics

P McIntyre

Correspondence to:

Dr G J Browne, Emergency

Department, New Children's

Hospital, PO Box 3515,

Parramatta, NSW 2124,

Australia.

Accepted 8 November 1996

Occult bacteraemia or bacteraemia due to pathogenic organisms without clinical evidence of focal infection was first highlighted as a clinical entity in young highly febrile children in Boston in the early 1970s. ${ }^{1}$ Since then, numerous studies have established that the likelihood of bacteraemia being present increases with increasing temperature and total white cell count and with decreasing age. ${ }^{2}$ Streptococcus pneumoniae is the causative organism in more than $80 \%$ of cases, with other pathogens such as Haemophilus influenzae type b, Neisseria meningitidis, Salmonella spp, and Streptococcus agalactiae occasionally found. ${ }^{12}$ The risk of meningitis after occult bacteraemia is primarily related to the causative organism; it is highest for $\mathrm{N}$ meningitidis, intermediate for $H$ influenzae type $\mathrm{b}$, and lowest for $S$ pneumoniae. ${ }^{4}$

With the decline and virtual disappearance of $H$ influenzae type b bacteraemia after immunisation against this organism in most industrialised countries, including the UK and Australia, ${ }^{5} S$ pneumoniae has become the overwhelming cause of occult bacteraemia. Opinion remains divided on whether empiric antibiotic treatment ${ }^{36}$ or careful observation without antibiotics ${ }^{7}$ is the most appropriate management for non-toxic febrile children with no evidence of focal infection who have risk factors ${ }^{2} 3$ for bacteraemia. Fever is a common presenting symptom in young children and excessive antibiotic use is of concern when empiric treatment is given frequently, both with regard to emerging antibiotic resistance and the potential to mask other diagnoses. $^{7}$

There are few data on occult bacteraemia in Australia $^{8}$ or the $\mathrm{UK}^{9}$ and, even in North America, management of potential occult bacteraemia in non-research settings has been little studied. ${ }^{10}$ In contrast to the US, where many young febrile children are managed in the offices of primary care paediatricians ${ }^{10}$ and children presenting to emergency departments are often socioeconomically disadvantaged, any children with acute symptoms in the UK and Australia are commonly seen in hospital emergency departments, either as a primary presentation or after referral from a general practitioner. A protocol to guide the management of this common problem of fever in children, 3 to 36 months of age, was developed in a busy urban general hospital children's emergency department in Sydney. The protocol aimed to minimise antibiotic use, while identifying as many cases of potential bacteraemia as possible. The implementation of this protocol was retrospectively evaluated with regard to the identification, treatment, and outcome of bacteraemia over a 12 month period.

\section{Methods}

Westmead Hospital, the major teaching hospital in the western Sydney region, has a separate paediatric emergency department that sees 15000 children under 12 years of age each year, of whom approximately $28 \%$ are admitted to hospital. There is an attached short stay facility for periods of observation up to 24 hours. ${ }^{11}$ Temperature was routinely recorded as part of the triaging process.

FEVER MANAGEMENT PROTOCOL

The protocol (fig 1) specified management of previously well children from 3 months to 36 


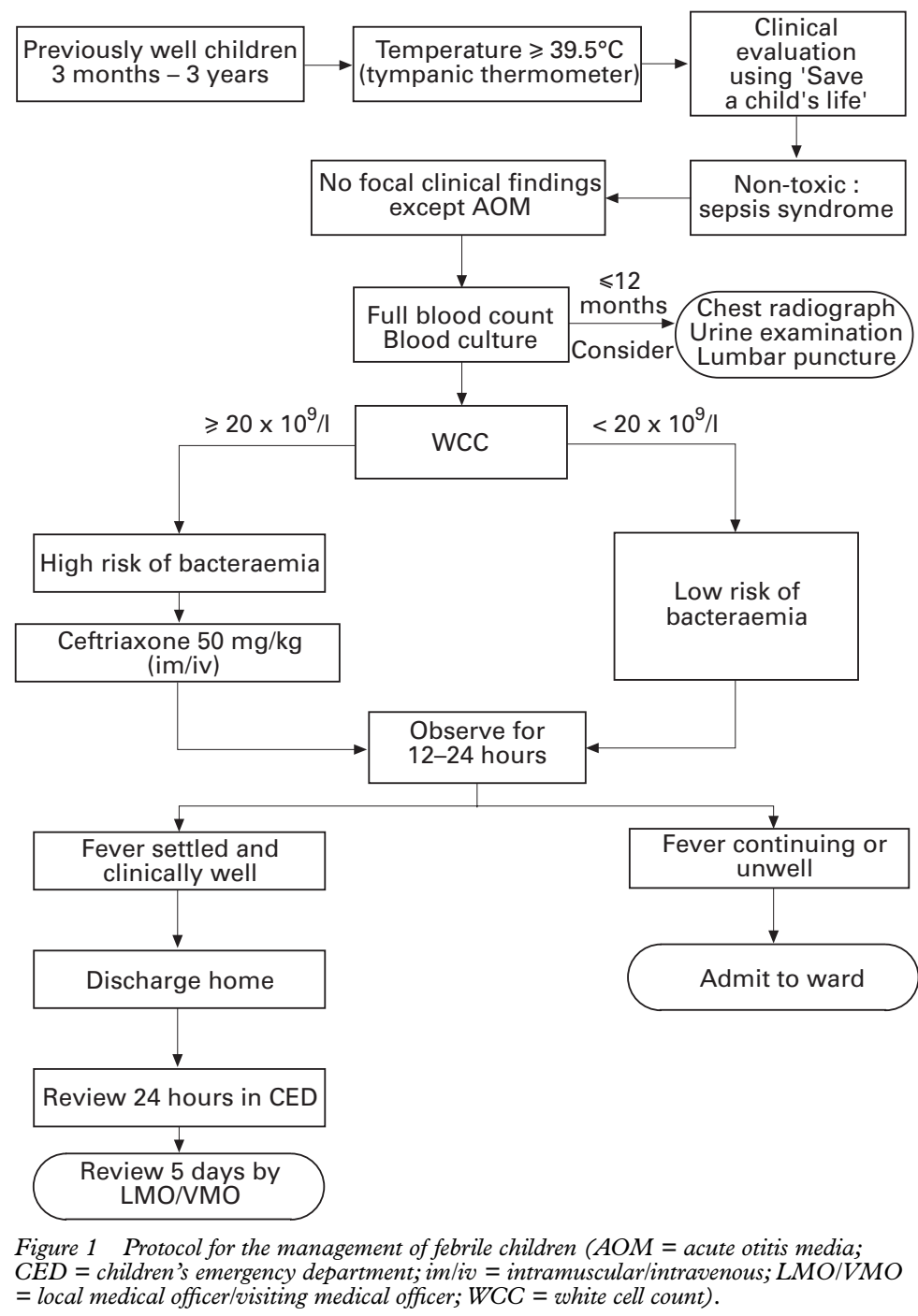

months of age who had a temperature of $\geqslant 39.5^{\circ} \mathrm{C}$ measured by tympanic thermometer and were not considered to be seriously unwell or 'toxic'. Nursing protocols specified that the highest of three temperature readings were taken by tympanic thermometer. If the nurse felt that the child was febrile and the tympanic temperature was $<38.5^{\circ} \mathrm{C}$, the temperature measurement was repeated with a regular digital thermometer in the axilla. Toxicity was defined as any of the clinical features lethargic appearance, signs of poor peripheral perfusion, hypoventilation, tachypnoea, or cyanosis. Lethargy was defined as poor or absent eye contact or failure of a child to recognise parents or to interact with persons or objects in the environment. Perfusion was assessed by evaluation of capillary return, and if prolonged beyond two seconds was regarded as abnormal. These are features of the 'sepsis syndrome' and if any of these were present at the initial evaluation the child was considered at risk of septicaemia and excluded from the study. ${ }^{12}$ An observation scale termed, 'Save a child's life' was used in the department to allow junior staff consistency in identifying children at risk of septicaemia. This scale was a modification of the Yale febrile child observation scale. ${ }^{13}$
Children with a temperature of $\geqslant 39.5^{\circ} \mathrm{C}$ who were not considered toxic on examination and had no findings of focal infection other than otitis media then had a full blood count and blood culture. Children with a total white cell count $\geqslant 20 \times 10^{9} / 1$ were considered to be at high risk of bacteraemia. ${ }^{11}$ They were given ceftriaxone $50 \mathrm{mg} / \mathrm{kg}$ intramuscularly or intravenously and admitted to the short stay ward. If fever continued or the child was considered to be more unwell by the attending medical officer, admission to a hospital bed was arranged. If the fever settled and there was clinical improvement, the child was discharged after 12 to 24 hours to return the next day. At follow up, the child was re-evaluated clinically, a second dose of ceftriaxone was administered and arrangements made for review by their general practitioner five days later. If any of a number of defined adverse events, as defined by the Australian College of Emergency Medicine, ${ }^{14}$ occurred during follow up, a critical incident form was completed. Parents were asked to return to hospital at any time if they felt their child had become more unwell.

\section{CASE ASCERTAINMENT}

Children fulfilling the case definition were ascertained in three stages. First, the emergency department log book was reviewed for the 12 month period 1 June 1994 to 31 May 1995 to exclude patients from outside the local referral area by postcode and outside the target age group. The medical records of the remaining children were then rapidly screened to exclude for any one of five common discharge diagnoses (asthma, croup, bronchiolitis, surgical diagnoses, and trauma) not thought relevant and children who did not have a temperature $\geqslant 39.5^{\circ} \mathrm{C}$ recorded during their stay in the emergency department. Detailed medical record review of these highly febrile children was then undertaken to exclude those who were admitted to hospital because a focus of infection was identified on initial assessment or there were features indicating toxicity. Children 3 to 36 months of age without identified focal infection who had a temperature of $\geqslant 39.5^{\circ} \mathrm{C}$ then formed the primary study population. Cross checking was carried out against other data bases available within the hospital (short stay annexe, financial services) to ensure complete ascertainment of cases with bacteraemia.

Adverse events were ascertained from the case notes at the second ( 24 hour) presentation and at the final five day follow up, all patients were given an adverse event form as developed by the Australian College for Emergency Medicine, ${ }^{14}$ to be completed at the discretion of the local medical officer. An adverse event was considered to have occurred if a child after discharge from the short stay ward developed evidence of septicaemia, focal infection, persisting fever, convulsion, or seizure and any problem thought to be related to the effects of the antibiotic such as diarrhoea. All patients returning to the emergency department without an appointment were referred to the attending emergency physician for evaluation. 


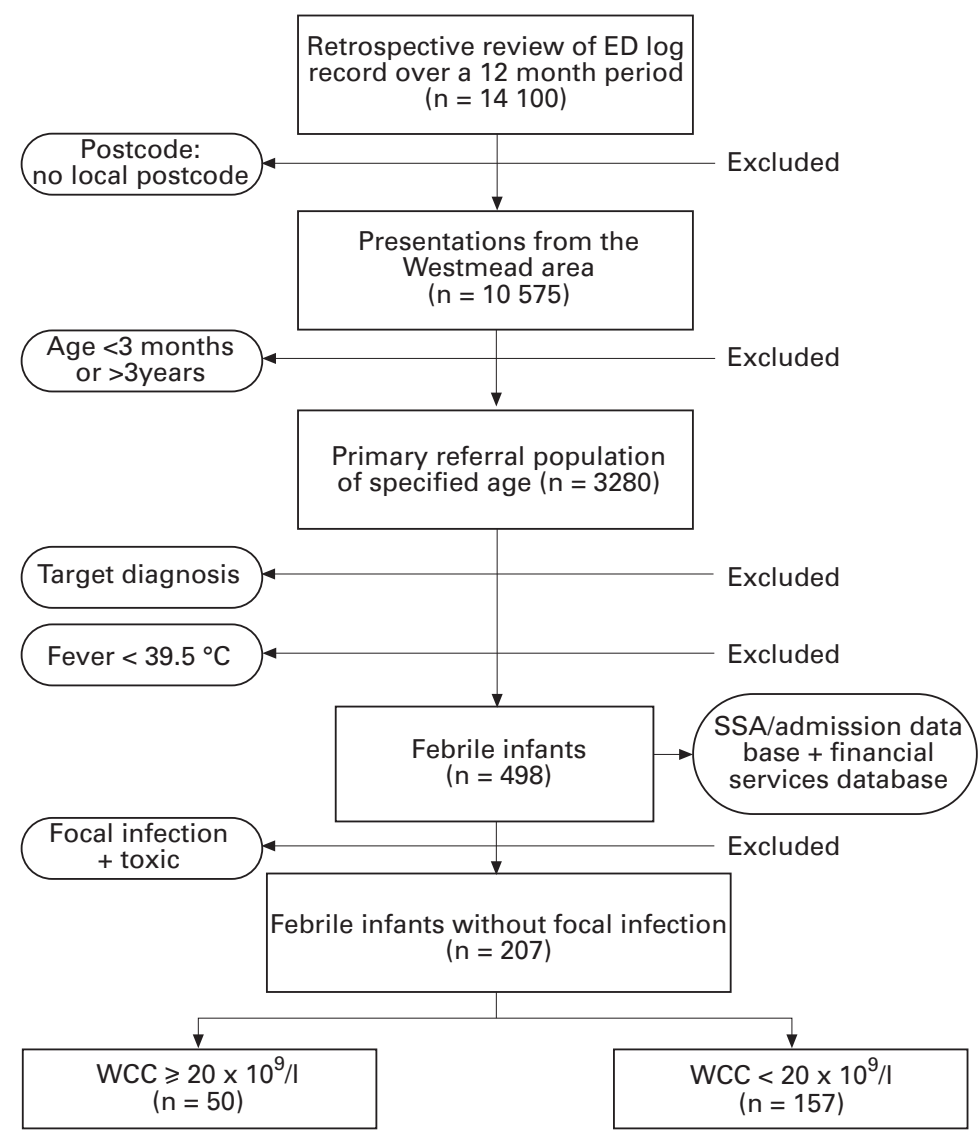

Figure 2 Case ascertainment (ED = emergency department; $S S A=$ short stay annexe; $W C C=$ white cell count).

Table 1 Study results

\begin{tabular}{llll}
\hline & \multicolumn{2}{l}{ Investigations performed (\%) } \\
\cline { 2 - 4 } $\begin{array}{l}\text { Age group } \\
\text { Full evaluation including } \\
\text { lumbar puncture }\end{array}$ & $\begin{array}{l}\text { Evaluation excluding } \\
\text { lumbar puncture }\end{array}$ & $\begin{array}{l}\text { White cell count and } \\
\text { blood culture only }\end{array}$ \\
\hline $\begin{array}{l}12 \text { months }(\mathrm{n}=72) \\
\begin{array}{l}12 \text { months }-2 \text { years } \\
(\mathrm{n}=80)\end{array}\end{array}$ & 57 & 37 & 6 \\
$>2$ years $(\mathrm{n}=55)$ & 12 & 54 & 11 \\
\hline
\end{tabular}

\section{Results}

The process of case ascertainment is shown in fig 2. From 14100 emergency department visits during the period of review, 10575 children were from postcodes in the primary referral area, of whom 3280 were in the target age group. There were 498 children with a temperature of over $\geqslant 39.5^{\circ} \mathrm{C}$ identified, of whom 207 were not initially admitted to hospital and had no focus of infection recorded on first assessment. All of these children had had a white cell count and a blood culture performed, of whom $50(24 \%)$ had a total white cell count $\geqslant 20 \times 10^{9} / 1$. Of these $50,10(20 \%)$ had a focus identified during observation which was urinary tract infection in seven, all of whom were less than 12 months of age and radiological changes consistent with pneumonia in three, one of whom was less than 12 months of age (fig 3).

Among the remaining 40 cases with no identified focus, 19 (48\%) were bacteraemic and 21 had negative blood cultures, with a presumptive diagnosis of viral infection. $S$ pneumoniae

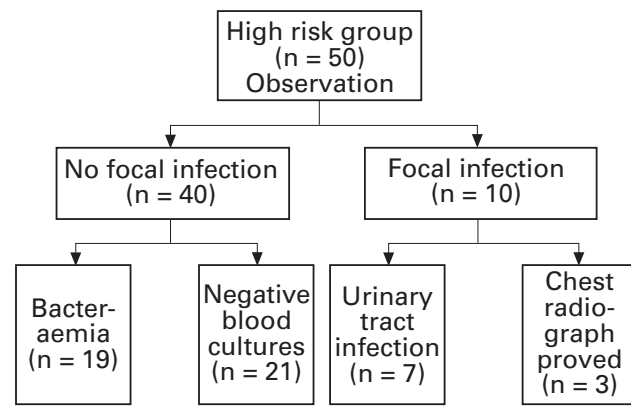

Figure 3 Results of children at high risk of bacteraemia.

was isolated in $16(84 \%)$ and $H$ influenzae in three bacteraemic cases, of whom six were under 12 months, 10 aged from 12 to 24 months, and three were more than 24 months. $H$ influenzae was the causative organism in two of those over 24 months of age and in one child of 18 months. Of those with a total white cell count $<20 \times 10^{9} / 1$, none of the 103 children (67\%) who had blood cultures performed were bacteraemic. Review of blood culture results during the study period did not reveal any episodes of bacteraemia not detected by record review.

As expected, investigations to detect a cause of fever were more extensive in younger febrile children (table 1). The 72 children under 12 months of age had complete blood count, blood cultures, urine examination, chest radiography and lumbar puncture in $57 \%$ of cases, significantly more than the $37 \%$ in the next age band of 12 months to 2 years (odds ratio 2.2, $95 \%$ confidence interval (CI) 1.2 to 4.2 ), which was in turn significantly greater than in those over 2 years $(6 \%$, odds ratio $10.4,95 \%$ CI 3.0 to 36.3). Children older than 1 year of age most commonly had only a full blood count and blood culture performed (48\% 2 to 3 years, $40 \% 1$ to 2 years) compared with $12 \%$ under 12 months.

All the 50 children with a total white cell counts $\geqslant 20 \times 10^{9} / 1$ and no clinically detected focus of infection had received antibiotic treatment before culture results were known. Of the 21 whose blood cultures were negative, $85 \%$ were given ceftriaxone and $15 \%$ were given oral amoxycillin, while all 19 children subsequently found to be bacteraemic had been treated with ceftriaxone. All 50 children returned for follow up. Three had unscheduled representations for fever, but none required admission to hospital. Two of three cases of $H$ influenzae bacteraemia were admitted to hospital after initial observation but were discharged without sequelae. None of the 157 children with white cell counts $<20 \times 10^{9} / 1$ were treated with ceftriaxone, with most having a discharge diagnosis of a presumed viral illness, although virologic investigations were not performed. No significant adverse events were documented at follow up.

\section{Discussion}

The controversy over management of fever without evident source in young children centres around whether outcome is improved by 
early detection and empiric treatment of bacteraemia, compared with treatment initiated because clinical signs of sepsis or a focus of infection have developed. ${ }^{7}$ Although there were three cases of $H$ influenzae type $\mathrm{b}$ bacteraemia in this series, $H$ influenzae type $\mathrm{b}$ has subsequently almost disappeared in Australia ${ }^{5}$ and other countries ${ }^{7}$ with established immunisation programmes. Occult bacteraemia is now almost always caused by $S$ pneumoniae. ${ }^{37}$ The reported natural history of occult pneumococcal bacteraemia is that approximately $30 \%$ of cases have become afebrile without specific treatment at follow up. ${ }^{213}$ The exact proportion of untreated bacteraemic children who later develop apparently new focal infections is uncertain. In a retrospective series from Boston, ${ }^{15}$ among 48 children with occult pneumococcal bacteraemia who did not initially receive antibiotic treatment, five $(10.4 \%)$ had new focal infections at follow up, compared with $15 / 344(4.4 \%)$ of bacteraemic children who had received empiric treatment. ${ }^{15}$ Persistent pneumococcal bacteraemia without focal infection was documented at follow up in $14.6 \%$ of untreated and $1.2 \%$ of treated patients. ${ }^{15}$ Meningitis was diagnosed at follow up in one $(2.1 \%)$ of the untreated patients and three $(0.9 \%)$ of the treated patients in Boston, ${ }^{15}$ a lower estimate than from a recent metaanalysis $(6 \%$ developing meningitis without antibiotic treatment compared with $1.5 \%$ if antibiotics have been given). ${ }^{16}{ }^{17}$

The prior probability of bacteraemia, which depends on the population studied, and the reliability of clinical assessment by observers of varying experience, are important parameters in decision making in the emergency department. Few studies have examined implementation of a policy for the recognition and management of occult bacteraemia in a routine primary care or emergency department setting. ${ }^{10}$ Tympanic measurements were used initially to identify children with temperatures $\geqslant 36.5^{\circ} \mathrm{C}$. Although there have been concerns with identification of lesser fevers by this method in young infants, ${ }^{18}$ tympanic temperature measurements are reliable in identifying temperatures $>38.5^{\circ} \mathrm{C}$ in this group. ${ }^{19}$ Tympanic measurements may also be falsely low in infants under 3 months of age, who were excluded from this study. ${ }^{20}$ In this study, triage by nursing and junior medical staff using a simple observation scale was successful in identifying febrile children requiring admission. Among children selected for outpatient assessment, a total white cell count of $20 \times 10^{9} / 1$ was a clinically useful cut off for detection of children at risk for bacteraemia.

The proportion of febrile children with very high white cell counts and the incidence of bacteraemia found in this study was similar to other studies using similar clinical cut offs in comparable populations. ${ }^{2315}$ The proportion of febrile children with a white cell count $>20$ $\times 10^{9} / 1(24.2 \%)$ resembled data in the same age group from a group of US emergency departments $^{15}(28.9 \%$, odds ratio $0.78,95 \%$ CI 0.5 to $1.1, p=0.2$ ) as did the overall proportion who were bacteraemic with a temperature $>39.5^{\circ} \mathrm{C}$ (Sydney $9.2 \%$ and the US $\left.11.6 \%{ }^{15}\right)$. Similarly, among those with both high fever and a total white cell count $>20 \times$ $10^{9} / 1$, the proportion with bacteraemia in Sydney $(38 \%)$ was higher but not significantly so than US emergency departments ${ }^{15}(29 \%$, odds ratio $1.7,95 \%$ CI 0.9 to $3.4, p=0.15)$. This suggests that the data from the Sydney study, although retrospective, and therefore more subject to ascertainment bias, are consistent with other emergency department populations and likely to be generalisable to elsewhere in Australia and the UK.

As blood cultures were taken in only $67 \%$ of cases with lower white cell counts in this series, some cases of bacteraemia may have been missed. Bacteraemia was not documented in any of the 103 children with white cell counts $<20 \times 10^{9} / 1$ who had blood cultures performed in the Sydney study (95\% CI 0 to $3.6 \%$ ) compared with $5.8 \%$ (95\% CI 3.6 to $8.8 \%$ ) from comparable US data. ${ }^{15}$ As these CIs for the expected proportion with bacteraemia overlap, the lack of bacteraemic cases in the present study may be due to either the relatively small sample studied or under ascertainment.

Increasing the threshold for empiric treatment to a white cell count of $20 \times 10^{9} / 1$, reduced the number of candidates for empiric treatment to 50, or 40 if those developing focal infection during observation are excluded. The number of patients necessary to treat for each case of bacteraemia detected, using these selective white cell count criteria, can be calculated as three (50/19) in Sydney, similar to the four (148/19) in a US emergency department population. ${ }^{15}$ This compares favourably with estimates of number needed to treat which can be derived from US data ${ }^{15}$ of $11(182 / 16)$ in those with white cell counts of between 15 and $20 \times 10^{9} / 1$ and $36(182 / 5)$ among those with a white cell count of $<15 \times 10^{9} / 1$. In most hospitals with paediatric emergency departments, a total white cell count is readily available from a finger prick blood sample within a short time, even after normal hours, making this a practical screening test. The predominance of pneumococcal bacteraemia would be expected to further enhance the sensitivity of the white cell count criteria adopted in the protocol used for this study, as most cases of bacteraemia with lower white cell counts are due to $H$ influenzae type $b^{7}$

The two options recommended by a recent expert US panel for management fever without evident source in children 3 to 36 months of age, is to treat all who have a temperature $>39.5^{\circ} \mathrm{C}$, or only those with fever $>39.5^{\circ} \mathrm{C}$ and a white cell count $>15 \times 10^{9} / 1 .^{3}$ This has been criticised, particularly in the post $H$ influenzae type $b$ era, because of excessive antibiotic use, reduced ability to observe children clinically, and the potential to miss diagnoses such as urinary tract infection. ${ }^{7}$ In contrast to many centres in the US, ${ }^{6715}$ our policy was to keep children selected for empiric treatment for a period of observation in the emergency department ${ }^{11}$ and to ensure that other investigations, such as an adequate urine specimen, ${ }^{7}$ had been obtained before treatment was 
started. The experience reported here suggests that a protocol using a staged approach to assessment of young children with high fever, to first identify those requiring admission to hospital and then define a subgroup at high risk for bacteraemia by total white cell count, is practical and safe for use in an emergency department with a large and changing staff. Relatively few children are given empiric antibiotic tratment and adequate follow up is assured. ${ }^{11}$ Furthermore, recent advances in blood culture technology mean that bacteraemia is often detected within 12 hours of cultures being processed by the laboratory. The blood cultures had signalled, indicating bacterial growth, in all 27 cases of pneumococcal bacteraemia at our hospital laboratory in the most recent nine month period. The crucial question of whether there is ${ }^{3613}$ or is not $^{7}$ overall benefit from empiric treatment and whether such treatment need be given parenterally remains. Additional data, preferably including a randomised trial of adequate size, are needed from settings where the pattern of health care and the populations of interest differ from the US, such as Australia and the UK.

1 McGowan JE, Bratton L, Klein JO, Finland M . Bacteremia in febrile children seen in a 'walk in' pediatric clinic. N Engl f Med 1973;288:1309-12.

$\mathcal{F}$ Med $1973 ; 288: 1309-12$.
2 Singer JI, Vest J, Prints A. Occult bacteremia and septicemia in the febrile child younger than two years. Emerg Med Clin in the febrile child younger that

3 Baraff LJ, Bass JW, Fleisher GR, et al. Practice guideline for the management of infants and children 0 to 36 months of age with fever without source. Pediatrics 1993;82:1-12.

4 Shapiro ED, Aaron NH, Wald ER, Chiponis D. Risk factors for development of bacterial meningitis among children with occult bacteremia. F Pediatr 1986;109:15-9.
5 McIntyre P, Chey T, Smith W. The impact of vaccination cintyre P, Chey $\mathrm{T}$, Smith W. The impact of vaccination againstHaemophilus influenzae type b dise

6 Fleisher GR, Rosenberg N, Vinci R, et al. Intramuscular versus oral antibiotic therapy for the prevention of meningitis and other bacterial sequelae in young, febrile children at risk for occult bacteremia. $\mathcal{F}$ Pediatr 1994;124:585-8.

7 Long S. Antibiotic therapy in febrile children: 'best-laid schemes'. F Pediatr 1994;124:585-8.

8 McIntyre P, Gray S, Vance J. Unsuspected bacterial infections in febrile convulsions. Med f A Ast 1990;152:183-

9 McIntyre P, Kennedy R, Harris F. Occult pneumococcal bacteraemia and febrile convulsions. BMF 1983;286:203-6.

10 Young PC. The management of febrile infants by primary care paediatricians in Utah: comparison with published practice guidelines. Pediatics 1995;95:623-8.

11 Browne GJ, Penna A. Short stay facilities: the future of efficient paediatric emergency services. Arch Dis Child 1996;74:309-13.

12 Lorens XS, McCracken GH. Sepsis syndrome and septic shock in pediatrics: current concepts of terminology, pathophysiology, and management. $\mathcal{F}$ Pediatr 1993;123: path-508.

13 McCarthy PL, Sharpe MR, Spiesel SZ, et al. Observation scales to identify serious illness in febrile children. Pediatrics 1982;70:802-9.

14 Vinen JD, Gaudry PL, Ashby R, et al. Critical incident monitoring study (CIMS) in emergency medicine - an interim report. Report to the Commonwealth Department of Human Services and Health. Canberra: Department of Health, 1994.

15 Harper MB, Bachur R, Fleisher GR. Effect of antibiotic therapy on the outcome of outpatients with unsuspected bacteraemia. Pediatr Inf Dis f 1995;14:760-7.

16 Bass JW, Steele RW, Wittler RR, et al. Antimicrobial treatment of occult bacteremia: a multicenter cooperative study. Pediatr Infect Dis $\mathcal{F}$ 1993;12:466-73.

17 Barraff LJ, Oslund S, Prather M. The effect of antimicrobial therapy on the probability of bacterial meningitis in children with fever without source treated as outpatients. Pediatrics 1993;92:140-3.

18 Vinci R, Garabedian C, Bauchner H. Accuracy of tympanic thermometry in a pediatric emergency department. $A m \mathcal{F}$ Dis Child 1990;144:429.

19 Chamberlain LM, Gandner J, Rubinoff JL, et al. Comparison of a tympanic thermometer to rectal and oral hermometers in a pediatric emergency department. Clin Pediatr (Phila) 1991;30:24-9.

20 Blis-Holtz J. Determination of thermoregulatory state in full term infants. Nurs Res 1993;42:204-7. 